Supplementary Information

\title{
Digitalized $\mathrm{K}^{+}$Occupancy in the Nanocavity \\ Holds and Releases Queues of $\mathrm{K}^{+}$in a Channel
}

Takashi Sumikama \& Shigetoshi Oiki

Department of Molecular Physiology and Biophysics,

University of Fukui Faculty of Medical Sciences, Fukui 910-1193, Japan 


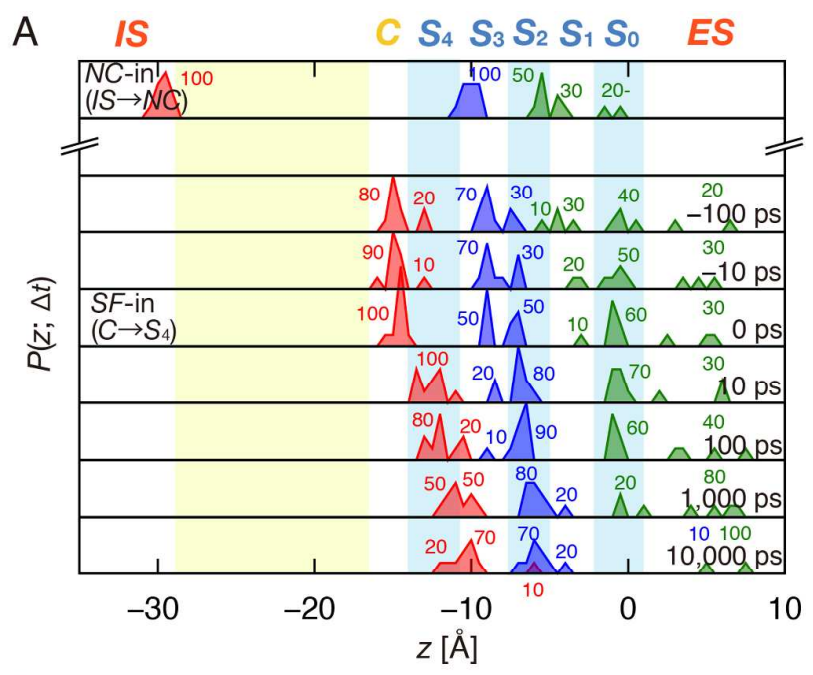

B

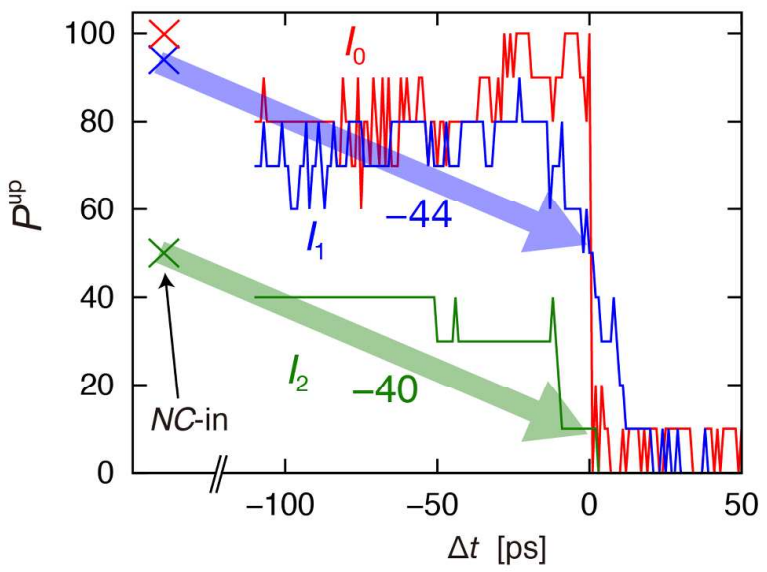

Figure S1. Time course of the ion distribution and the $P^{\text {up }}$ values at $+200 \mathrm{mV}$. Ion trajectories were collected as follows. During permeation at $+1000 \mathrm{mV}$, the membrane potential was changed to $+200 \mathrm{mV}$. After several ions permeated the channel, the ion trajectories were served for the event-oriented analysis. If the initial configurations were substantially different from those at the stationary state, the trajectories were omitted from the analyses. (A) $S F$-in oriented distribution. The red, blue, and green distributions are for $I_{0}, I_{1}$, and $I_{2}$, respectively. The numbers next to the distribution peaks denote the probability (\%) of each ion being at a discrete site. The ion distributions at the $N C$-in moment are also shown in the top panel. (B) $P^{\text {up }}$ values around the $S F$-in moment. When the $S F$-in event occurred, $I_{1}$ did not jump; instead, this ion gradually shifted outward. $P_{I 0}{ }^{\text {up }}=P\left(I_{0}{ }^{I S}\right)+P\left(I_{0}{ }^{N C}\right)(\mathrm{red}) ; P_{I 1}{ }^{\text {up }}=P\left(I_{1}{ }^{S 4}\right)$ $+P\left(I_{1}^{S 3}\right)$ (blue); $P_{I 2}{ }^{\text {up }}=P\left(I_{2}^{S 2}\right)+P\left(I_{2}^{S 1}\right)$ (green). The time course of $P^{\text {up }}$ provides the flux for the corresponding ion. 


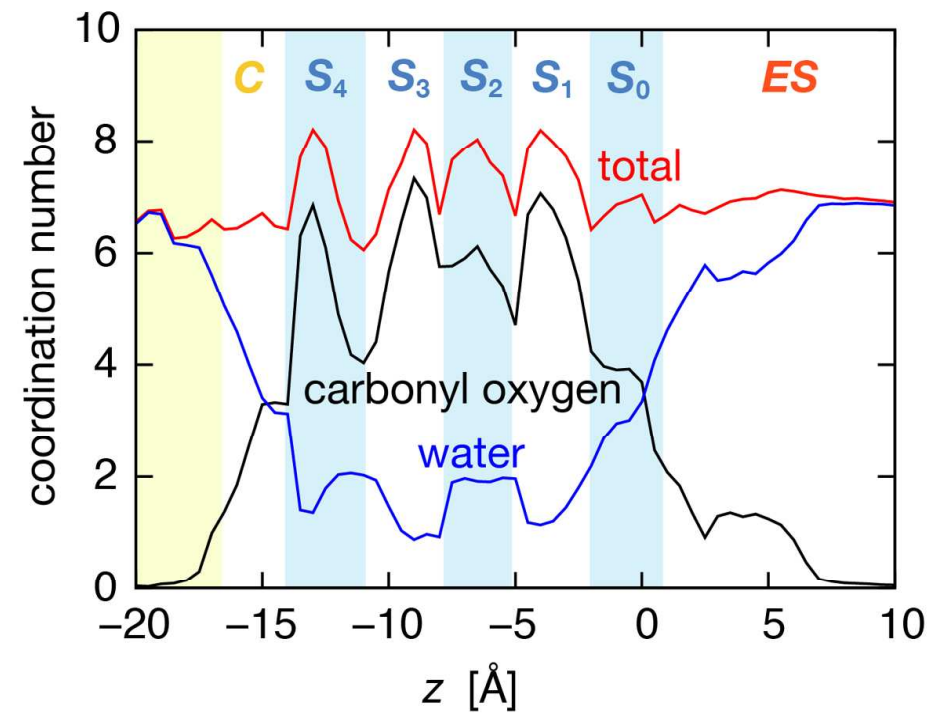

Figure S2. Coordination numbers for a $\mathrm{K}^{+}$ion in the pore. The average numbers of carbonyl, water, and total oxygen atoms within the distance of $R_{\mathrm{h}}$ (the first minimum in the radial distribution function of $\mathrm{K}^{+}$in water) were indicated as a function of the location of a $\mathrm{K}^{+}$ion along the pore. 


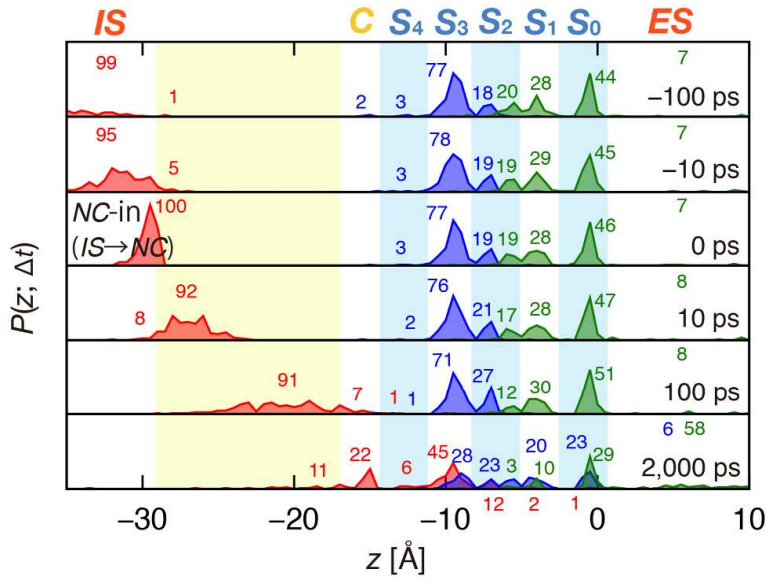

Figure S3. $N C$-in oriented distribution at $+1000 \mathrm{mV}$. $\Delta t=0$ was chosen as the moment when $I_{0}$ enters the $N C$ from the $I S$. The red, blue, and green distributions are for $I_{0}, I_{1}$, and $I_{2}$, respectively. The numbers next to the distribution peaks denote the probability (\%) of each ion being at each site. The ion distribution panel at $\Delta t=0$ is identical to that in the top row of Fig. $2 \mathrm{C}$ in the main text. The ion distributions in the $S F$ begin to shift outward after the $N C$-in moment (positive $\Delta t$ ), whereas the distributions are mostly immobilized for negative $\Delta t$. Thus, the $N C$-in event of $I_{0}$ drives the outward motion of $I_{1}$ and $I_{2}$.

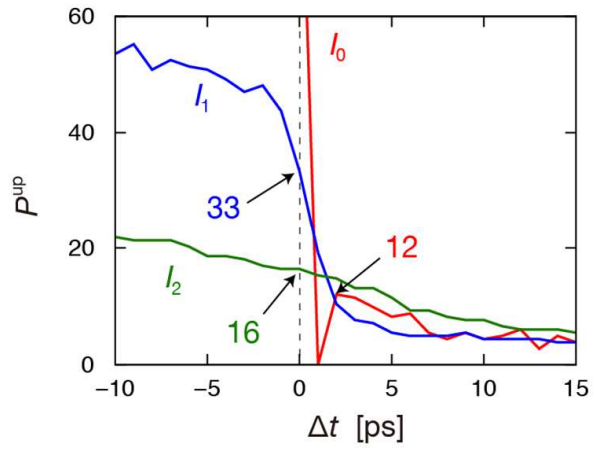

Figure S4. Close-up view of $P^{\text {up }}$ (the probabilities of ions occupying the upstream sites; Fig. $3 \mathrm{~A}$ in the text) around the $S F$-in moment. The red, blue, and green lines represent $P_{I 0}$ up, $P_{I 1}{ }^{\text {up }}$, and $P_{I 2}{ }^{\text {up }}$, respectively, in percentage. After the $S F$-in event, a fraction of $I_{0}(12 \%)$ returned to the $N C$ (re-crossing) and then gradually shifted outward. A fraction of $I_{1}$ jumped outward simultaneously with the $I_{0}$ jump at the $S F$-in moment, and gradual outward shifts similar to those of $I_{0}$ were subsequently seen. For $I_{2}$, no jump motion was observed around $\Delta t=0$. 

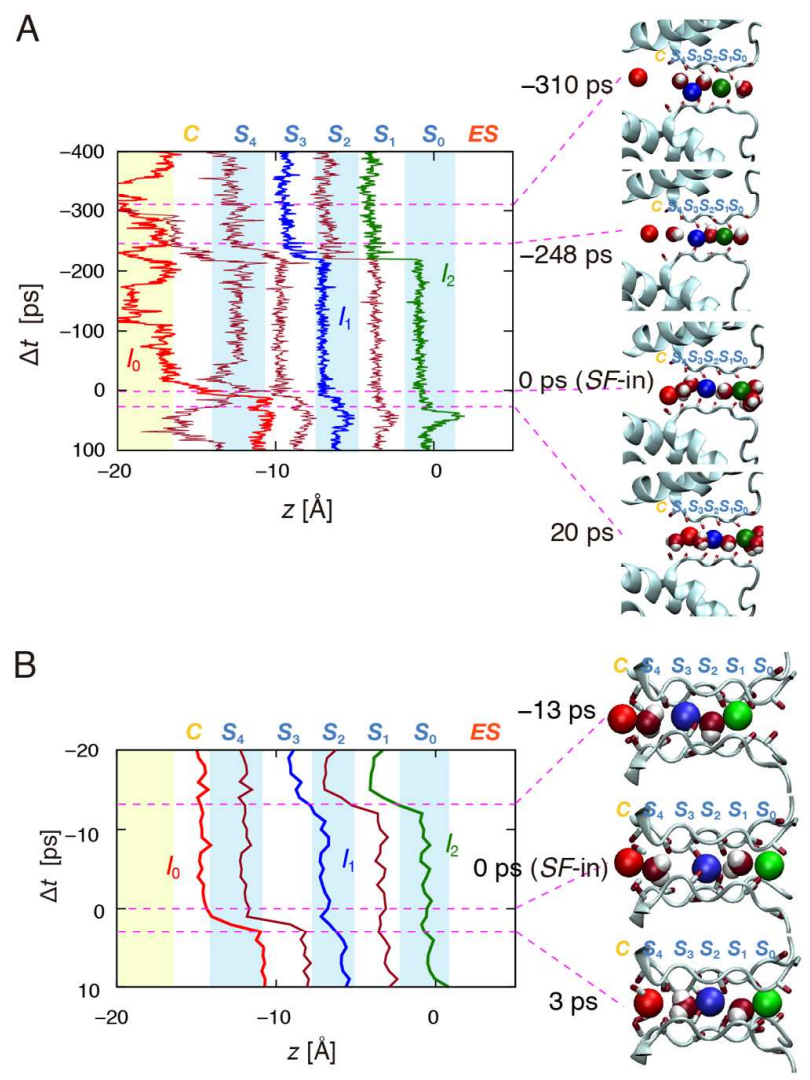

Figure S5. An event ( $S F$-in)-oriented trajectory of $I_{0}$ (red), $I_{1}$ (blue) and $I_{2}$ (green) with water molecules (brown) that intercalate the ions and snapshot images at defined times. The simulation trajectories indicate that various mechanistic routes exist. (A) Early preceding motions of $I_{1}$ and $I_{2}$. Trajectories were traced from $\Delta t=-400 \mathrm{ps}$, and $I_{0}$ was located in the $N C$ positions except for the $C$ site until $\Delta t \sim 0$. The outward shifts of $I_{1}$ and $I_{2}$ occurred at $\Delta t \sim$ -200 ps. When $I_{1}$ moved to $S_{2}$, the $S_{4}$-located water molecule moved to the $S_{3}$ site, leaving a vacant space at $S_{4}$ (typically for a few ps, at most a few tens of ps) until either $I_{0}$ moved in or a water in the $N C$ filled $S_{4}$. The snapshot at the $S F$-in shows the latter case, in which two water molecules occupied $S_{4}$ and $S_{3}$. After the $S F$-in moment, the water in $S_{4}$ was replaced by $I_{0}$. This is an infrequent process. The snapshots (the same as Fig. $3 \mathrm{C}$ in the main text) show the single-file configurations of ion and water. (B) Late preceding motion of $I_{1}$ and $I_{2} . I_{0}$ remained at $C$ from $\Delta t=-20 \mathrm{ps}$; at $\Delta t=-13 \mathrm{ps}, I_{1}$ and $I_{2}$ shifted downstream, leaving a vacant space at $S_{3}$ for more than $10 \mathrm{ps}$. The $S_{4}$-located water molecule coordinated the $C$-located $I_{0}$. Around the $S F$-in moment $(\Delta t \sim 0)$, the water molecule at $S_{4}$ moved to $S_{3}$ together with $I_{0}$. This is the major process. See also Movies. S1-3. 


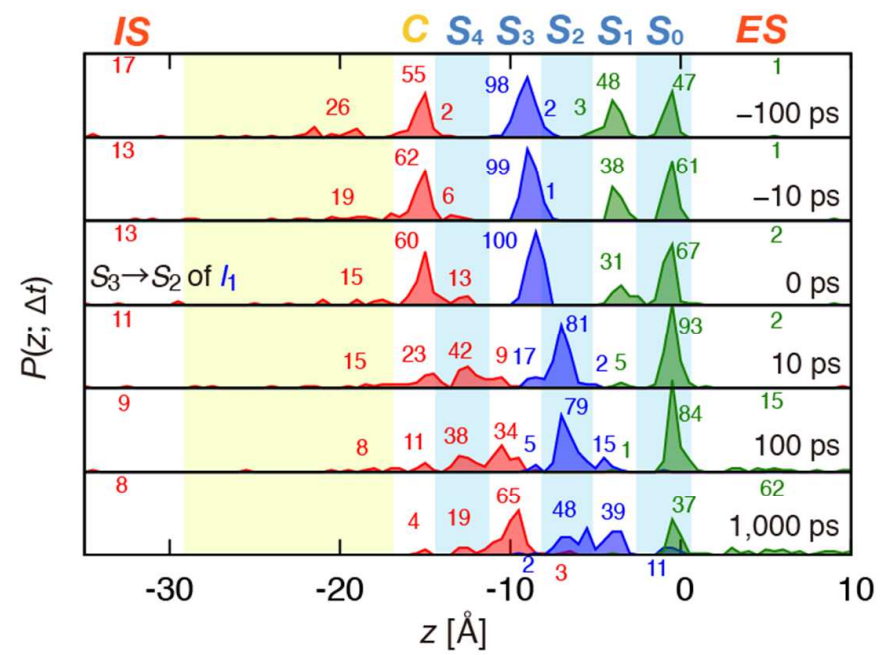

Figure S6. The ion distribution before and after the transition of $I_{1}$ from $S_{3}$ to $S_{2} . \Delta t=0$ was chosen as the moment when $I_{1}$ jumped from $S_{3}$ to $S_{2}$. The red, blue, and green distributions are for $I_{0}, I_{1}$, and $I_{2}$, respectively. The numbers next to the distribution peaks denote the probability (\%) of each ion being at a discrete site. At $-100 \mathrm{ps}<\Delta t<0, I_{2}$ gradually shifted outward from $S_{1}$ to $S_{0}$, whereas $I_{0}$ slightly accumulated at $S_{4}$. At positive $\Delta t, I_{2}$ was immediately evacuated towards $S_{0}$ and $E S$, and the distribution of $I_{0}$ widened.

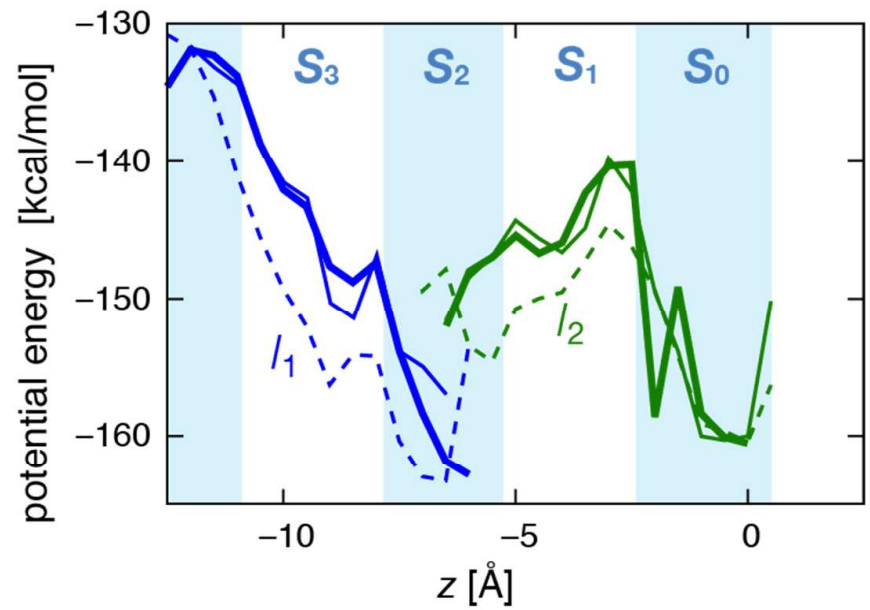

Figure S7. The potential energy profile as a function of $z$. The blue line is for $I_{1}$ and the green for $I_{2}$. Thin lines are the same as in Fig. 4A. The thick lines are the energy profiles in the period from the $N C$-in moment to the moment when $I_{0}$ reaches the $C$ site. 


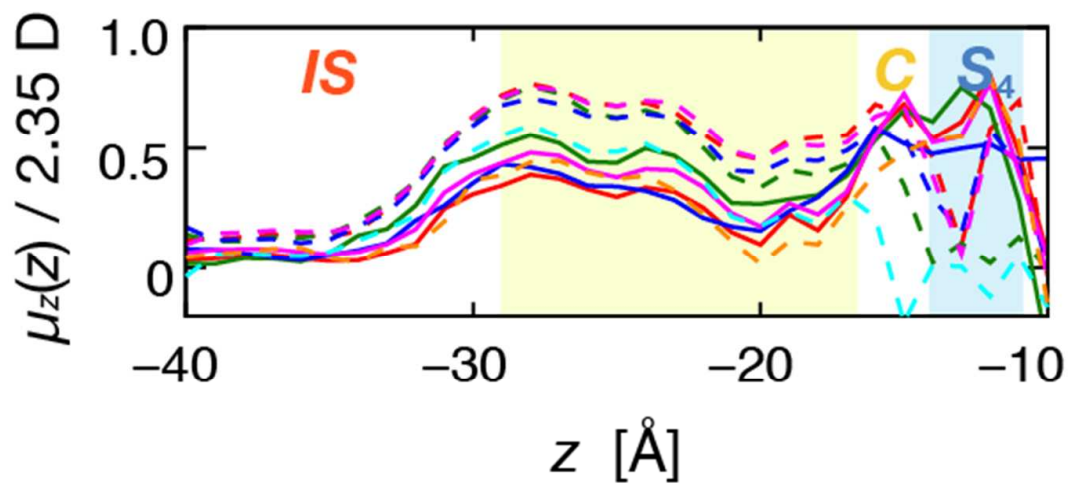

Figure S8. The $z$ component of the mean water dipole moment, $\mu_{z}(z)$. The red lines are for the TIP3P model (the same as Fig. 4B in the text). The blue, green, and magenta lines are those when the ff99SB force field was used for the Kv1.2 channel (column \#3 in Tab. S1), when the SPC/E water model was examined (column \#4 in Tab. S1), and when the electric field was applied to the entire system (column \#5 in Tab. S1), respectively. The dipole moment is normalized. The dashed lines indicate $\mu_{z}(z)$ in the ion-unoccupied period in $N C$, and the solid lines are for the ion-occupied period. The cyan line is $\mu_{z}(z)$ when the voltage is not applied, and the orange is $\mu_{z}(z)$ when the charges on the pore helix are turned off. 

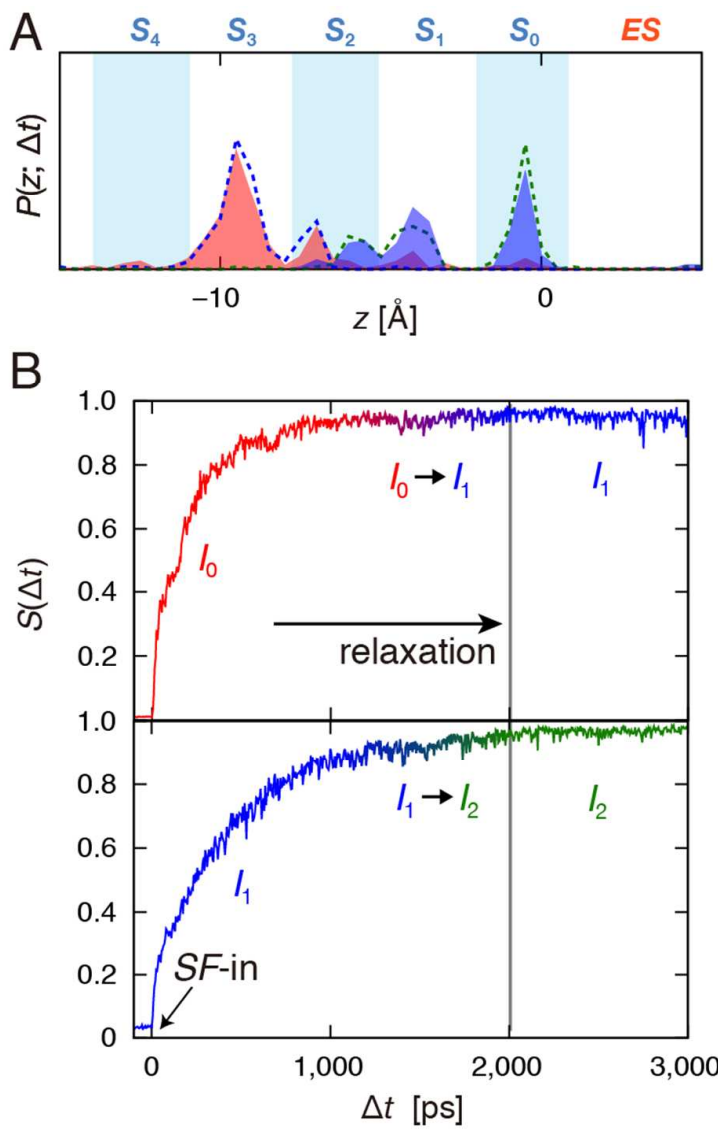

Figure S9. The relaxation process from the $S F$-in moment. (A) Superimposed ion distributions at $\Delta t=2,000 \mathrm{ps}$ from the $S F$-in moment $\left[P(z ; \Delta t=2,000)\right.$; the red area is for $I_{1}$, and the blue for $\left.I_{2}\right]$ and at the $N C$-in moment $\left[P_{N C}(z)\right.$; the blue broken line is for $I_{1}$, and the green one is for $\left.I_{2}\right]$. The nearly overlapping distributions at $\Delta t=2,000 \mathrm{ps}$ and at the $N C$-in time indicate that after the relaxation processes are completed, the ion distributions are maintained until the next ion enters the $N C$; this time is called the ion-immobilized period. (B) Degree of distribution overlap between $P(z ; \Delta t)$ and $P_{N C}(z)$ as a function of $\Delta t$. The overlap of the two distributions, $S(\Delta t)$, is defined as $S(\Delta t)=1 / \mathrm{AB} \int P_{N C}(z) P(z ; \Delta t) d z$, where $\mathrm{A}=\left(\int\right.$ $\left.P^{2}{ }_{N C}(z) d z\right)^{1 / 2}$ and $\mathrm{B}=\left(\int P^{2}(z ; \Delta t) d z\right)^{1 / 2}$. If two distributions are identical, then $S(\Delta t)=1$; otherwise, $S(\Delta t)=0$. The line graded from red to blue (upper panel) is $S(\Delta t)$ between $P_{I 0}(z ; \Delta t$ $=2,000 \mathrm{ps}$ ) and $P_{N C}(z)$ of $I_{1}$. The line graded from blue to green (lower panel) is $S(\Delta t)$ between $P_{I 1}(z ; \Delta t=2,000 \mathrm{ps})$ and $P_{N C}(z)$ of $I_{2}$. The $P_{I 0}(z ; \Delta t=2,000 \mathrm{ps})$ and $P_{I 1}(z ; \Delta t=2,000 \mathrm{ps})$ are very close to $P_{N C}(z)$ of $I_{1}$ and $P_{N C}(z)$ of $I_{2}$, respectively. That is, $I_{0}$ and $I_{1}$ respectively replace the positions of $I_{1}$ and $I_{2}$ in the former permeation event. 


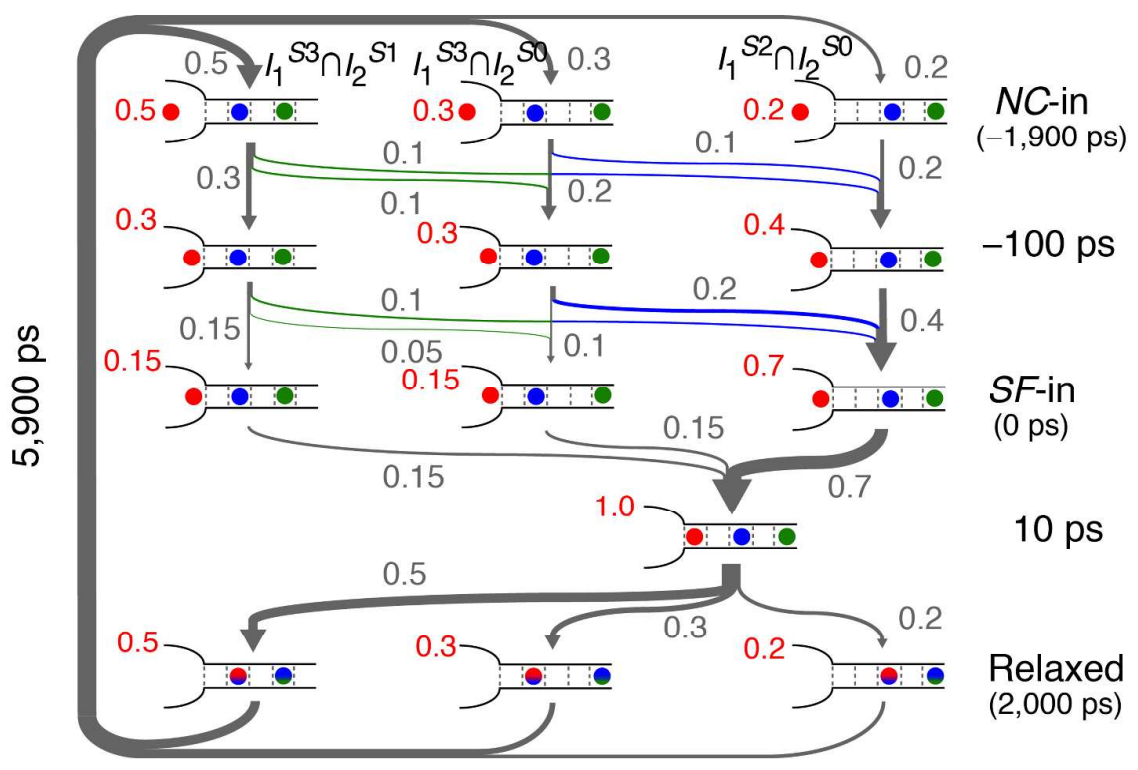

Figure S10. Detailed ion occupancy states and the transitions among them in the ion-mobile phase. The ion occupancies in the $N C$ (left wide pore) and the $S F$ (and right narrow pore for $S_{4}$ $-S_{0}$ ) at $\Delta t$ from the $S F$-in moment are schematically shown (red sphere for $I_{0}$, blue for $I_{1}$, and green for $I_{2}$ ). All the joint probabilities of the ion locations and their transitions were extracted from the MD data via event-oriented trajectory sampling. The red numbers are the probabilities that $I_{1}$ and $I_{2}$ occupy the $S F$ for given $I_{0}$ locations. The states with a probability less than 0.1 are omitted for the clarity. The thickness of the arrows connecting the states reflects the width of the pathways, which are labeled with gray numbers. The blue and green arrows indicate the pathways where $I_{1}$ and $I_{2}$, respectively, shift outward before the $S F$-in moment. 


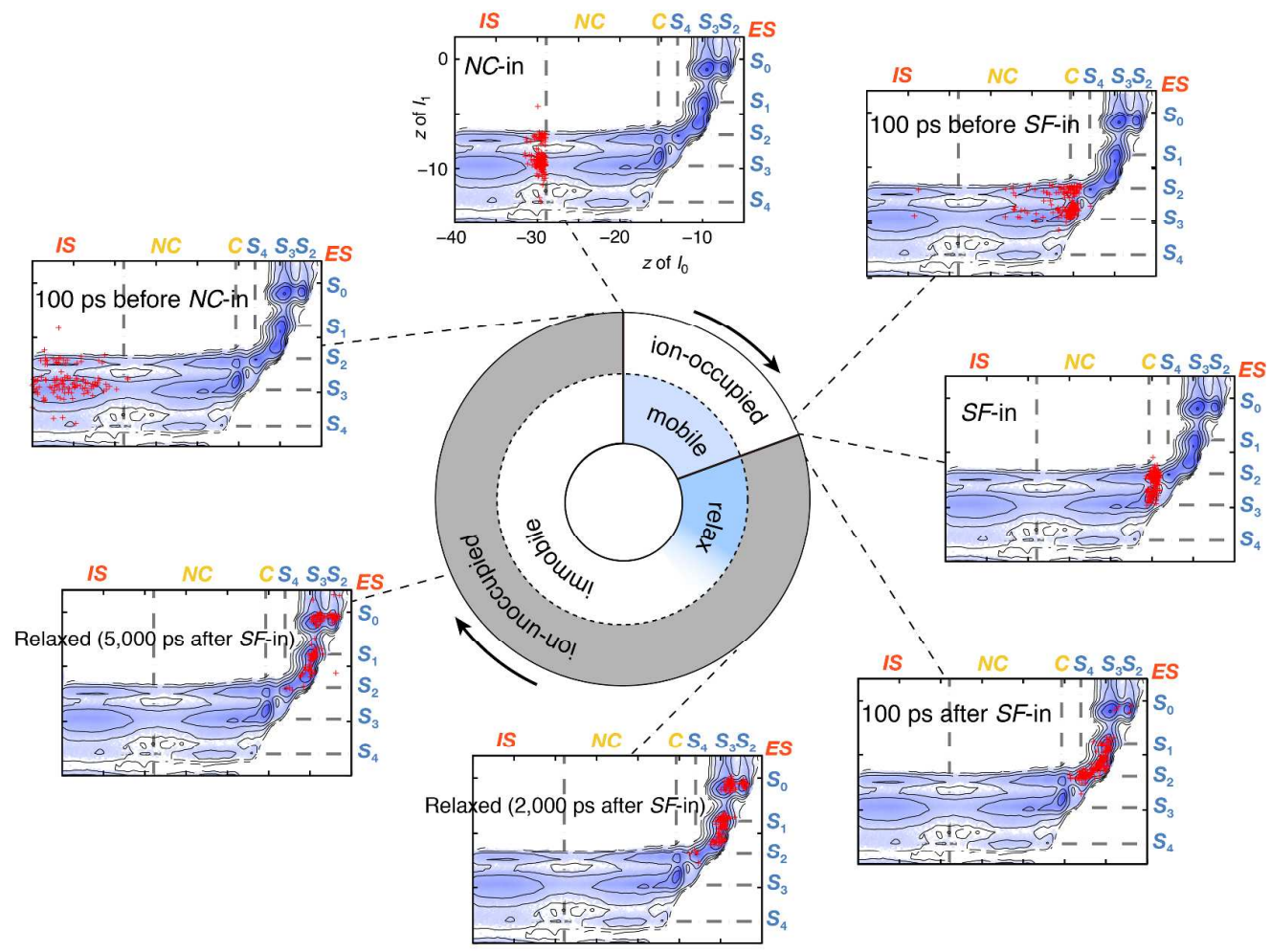

Figure S11. Progression of $I_{0}$ and $I_{1}$ along the 2-dimensional free energy surface during one permeation cycle. The 2-D free energy surface as a function of the $z$ positions of $I_{0}\left(z_{I 0}\right.$, horizontal axis) and $I_{1}\left(z_{I 1}\right.$, vertical axis), $F\left(z_{I 0}, z_{I 1}\right)$, was calculated from all the trajectories of the MD simulations using following equation; $F\left(z_{I 0}, z_{I 1}\right)=-k_{\mathrm{B}} T \ln \left(P\left(z_{I 0}, z_{I 1}\right)\right)$, where $k_{\mathrm{B}}$ and $T$ are the Boltzmann constant and the temperature, respectively. $P\left(z_{I 0}, z_{I 1}\right)$ is the conditional probability or the 2-D distribution given by $1 / T_{\text {sim }} \int P\left(z_{I 0}, z_{I 1} ; \Delta t\right) \Delta t$, where $T_{\text {sim }}$ and $\Delta t$ are the simulation time and the time from the $S F$-in moment (or the $N C$-in moment), respectively. $P\left(z_{I 0}, z_{I 1} ; \Delta t\right)$ is the joint probability at $\Delta t$ obtained by combining $P_{I 0}(z ; \Delta t)$ and $P_{I 1}(z ; \Delta t)$. The names of the sites are indicated as well as the $z$ coordinate. The free energy level is indicated with contour lines with an energy interval of $1 \mathrm{kcal} / \mathrm{mol}$; for the blue-filled contours, darker blue indicates the lower free energy. Around the cyclic phase diagram, progressions of ions on the 2-D free energy surface are shown with red dots that give the value of $P\left(z_{I 0}, z_{I 1} ; \Delta t\right)$ at the corresponding moment on the cyclic diagram. As shown on the surface, the $N C$-in event and the $S F$-in event are shown as clustered dots on the energy barrier; these events are the transition states in the cyclic reaction. Correlated motions of $I_{0}$ and $I_{1}$ start from the $N C$-in moment and continue until the relaxation. Afterward, the ions are immobilized. 

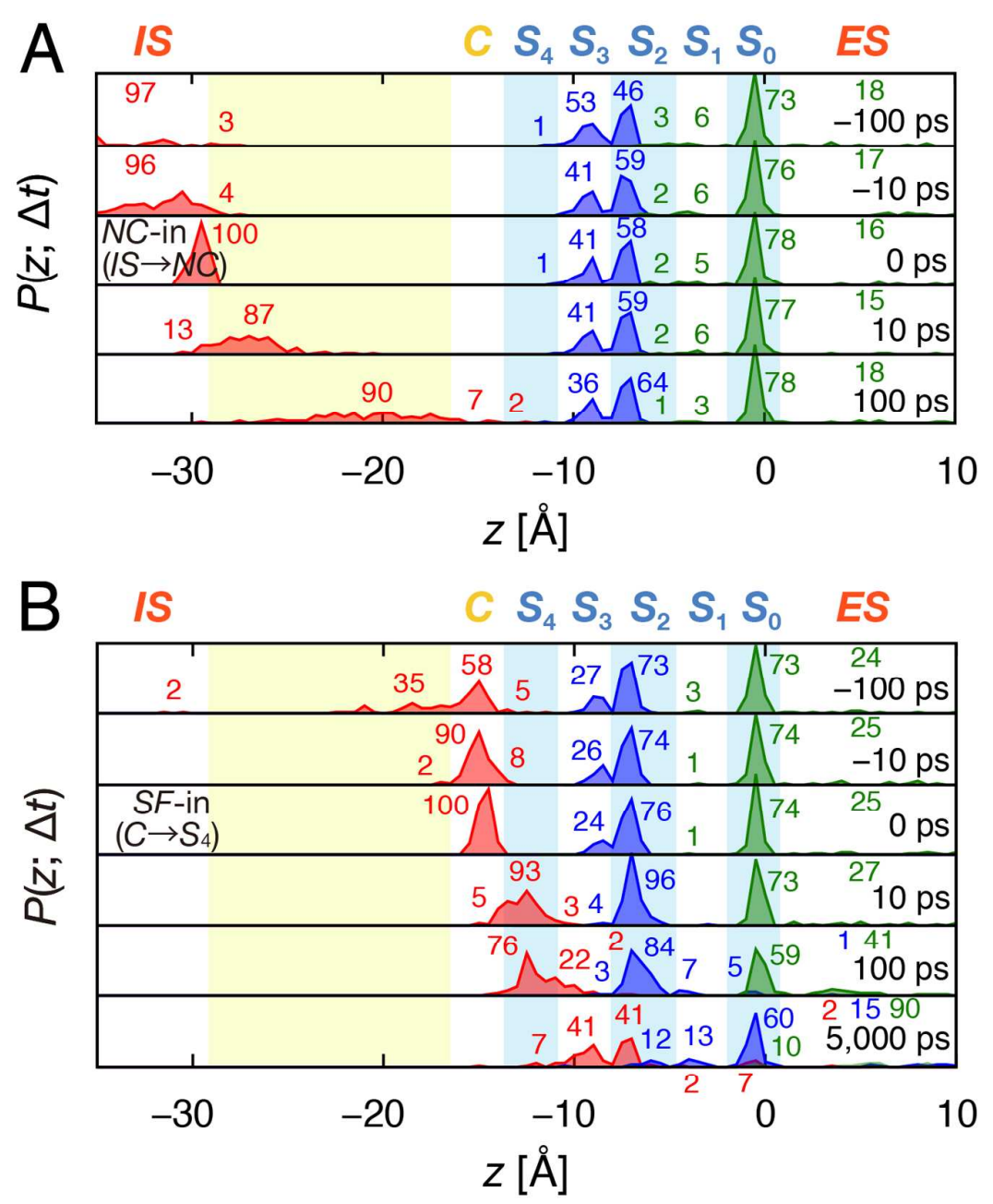

Figure S12. Event oriented distributions at $0.05 \mathrm{M} \mathrm{K}^{+}$. The red, blue, and green distributions are for $I_{0}, I_{1}$, and $I_{2}$, respectively. The numbers next to the distribution peaks denote the probability (\%) of each ion being at a discrete site. (A) $N C$-in oriented distribution. (B) $S F$-in oriented distribution. 


\begin{tabular}{|c|c|c|c|}
\hline IS & & $C S_{4} S_{3} S_{2} S_{1} S_{0}$ & ES \\
\hline 96 & 1 & $8 \quad 11^{70} \Omega^{6} 921^{38}$ & $\begin{array}{c}1 \\
-100 \mathrm{ps}\end{array}$ \\
\hline 973 & 1 & $3 \quad 9^{78} \wedge^{2} 918 \wedge^{44}$ & $\begin{array}{c}1 \\
-10 \mathrm{ps}\end{array}$ \\
\hline $\begin{array}{l}N C \text {-in } / 100 \\
(I S \rightarrow N C)\end{array}$ & 1 & $3 \quad 8^{78} \wedge^{2} 1017 \wedge^{45} \wedge^{35}$ & ${ }^{1} 0 \mathrm{ps}$ \\
\hline $11 \underbrace{89}$ & 1 & $3 \quad 6^{78} \wedge^{3} 1316 \wedge^{45} \wedge^{35}$ & ${ }^{1} 10 \mathrm{ps}$ \\
\hline & 891 & $10116^{73} \bigwedge^{2} 199 \bigwedge^{47} \Lambda^{41}$ & $\begin{array}{r}1 \\
100 \mathrm{ps} \\
\end{array}$ \\
\hline-30 & -20 & $\begin{array}{l}-10 \\
z[\AA \AA\end{array}$ & 10 \\
\hline
\end{tabular}

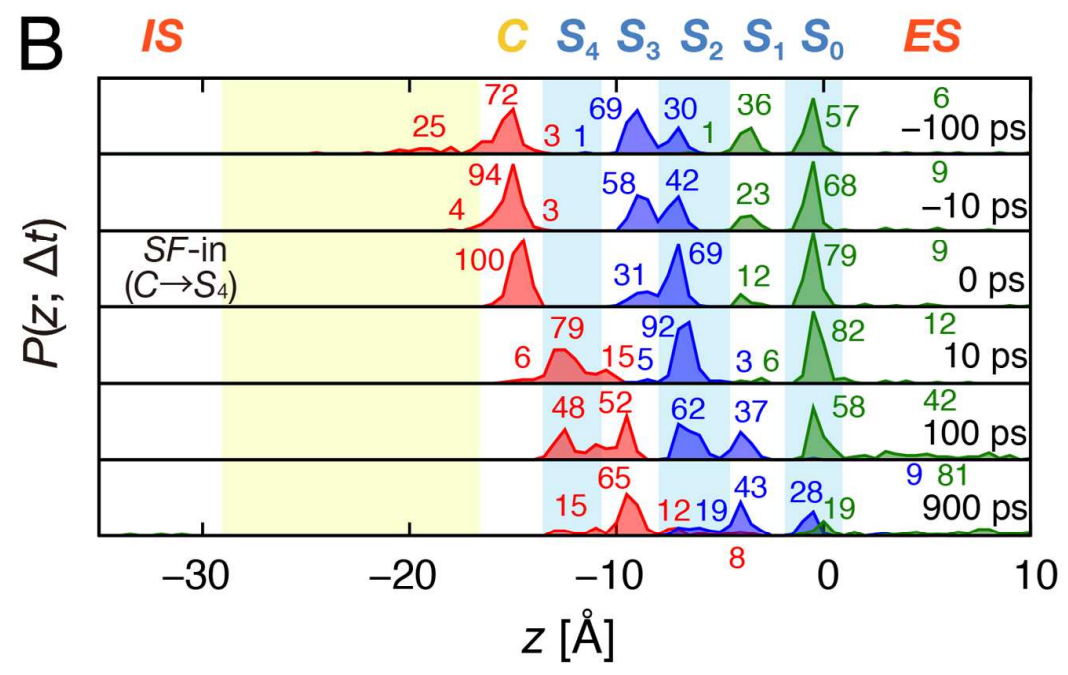

Figure S13. Event-oriented distributions at $0.54 \mathrm{M} \mathrm{K}^{+}$. The red, blue, and green distributions are for $I_{0}, I_{1}$, and $I_{2}$, respectively. The numbers next to the distribution peaks denote the probability (\%) of each ion being at a discrete site. (A) $N C$-in oriented distribution. (B) $S F$-in oriented distribution. 

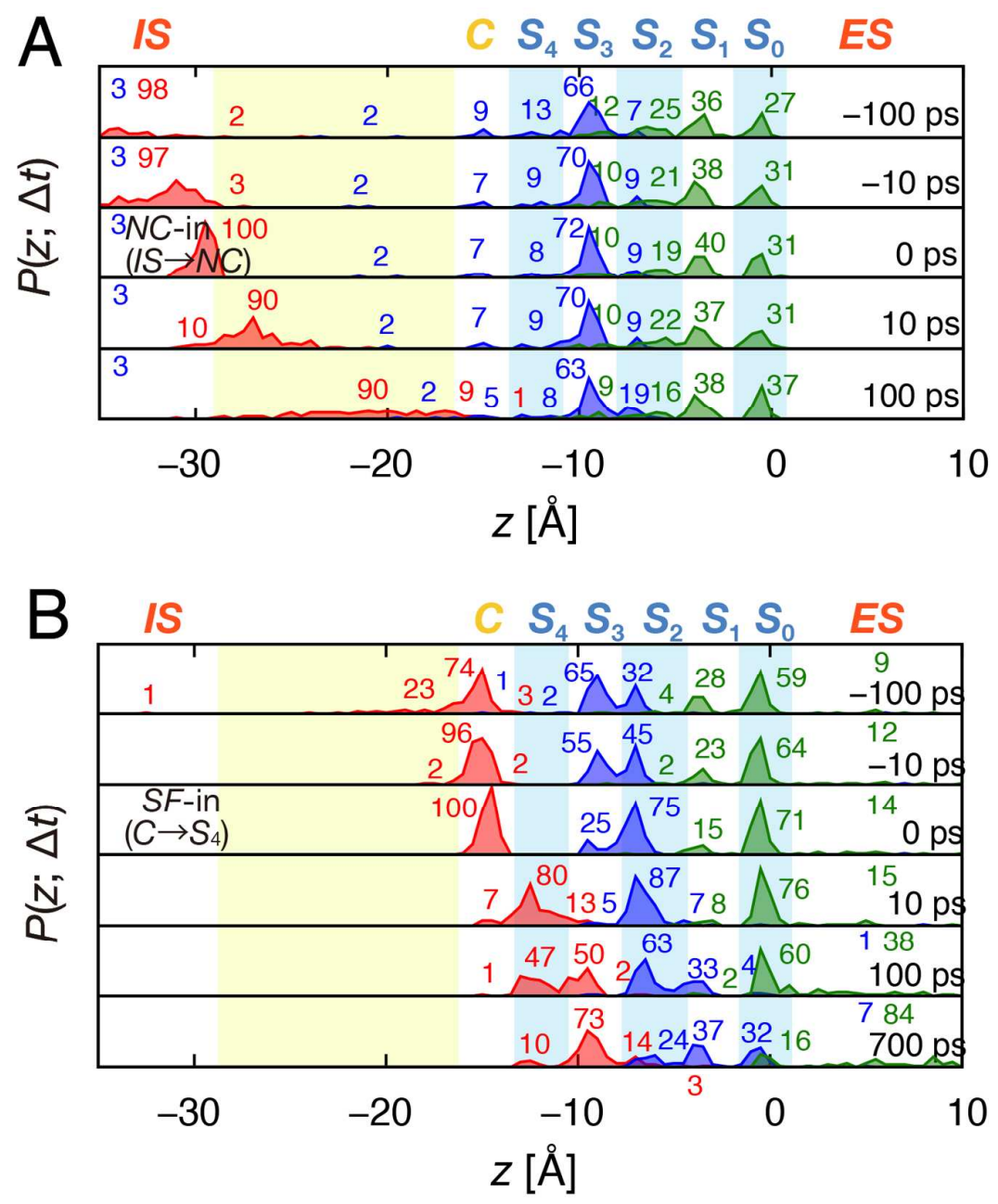

Figure S14. Event-oriented distributions at $0.99 \mathrm{M} \mathrm{K}^{+}$. The red, blue, and green distributions are for $I_{0}, I_{1}$, and $I_{2}$, respectively. The numbers next to the distribution peaks denote the probability (\%) of each ion being at a discrete site. (A) $N C$-in oriented distribution. (B) $S F$-in oriented distribution. 

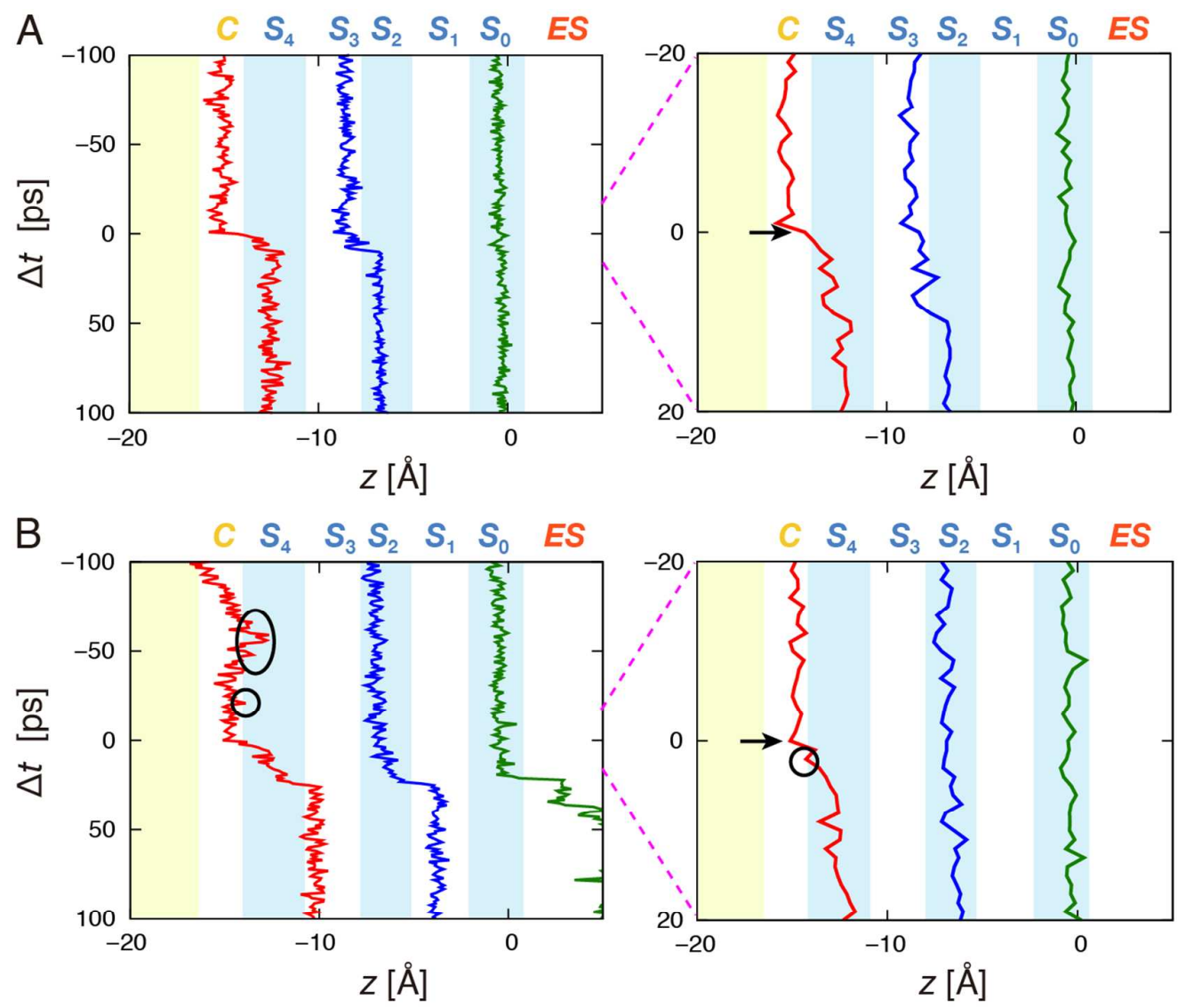

Figure S15. $S F$-in oriented trajectories. The red, blue, and green lines show the trajectories of $I_{0}, I_{1}$, and $I_{2}$, respectively. Black arrows indicate $S F$-in moments $(\Delta t=0)$. (A) Trajectories without re-crossing. (B) Trajectories with re-crossings shown by circles. 
Table S1. MD simulations at $0.14 \mathrm{M}$ under various simulation conditions.

\begin{tabular}{|c|c|c|c|c|c|}
\hline & $\# 1$ & $\# 2$ & $\# 3$ & \#4 & $\# 5$ \\
\hline $\begin{array}{c}\text { force fields for } \\
\text { water and } \\
\text { Kv1.2 }\end{array}$ & $\begin{array}{l}\text { TIP3P for } \\
\text { water and } \\
\text { ff94 for } \\
\text { Kv1.2 }\end{array}$ & $\begin{array}{l}\text { TIP3P for } \\
\text { water and } \\
\text { ff94 for } \\
\text { Kv1.2 }\end{array}$ & $\begin{array}{c}\text { TIP3P for } \\
\text { water and } \\
\text { ff99SB for } \\
\text { Kv1.2 }\end{array}$ & $\begin{array}{l}\text { SPC/E for } \\
\text { water and } \\
\text { ff99SB for } \\
\text { Kv1.2 }\end{array}$ & $\begin{array}{l}\text { TIP3P for } \\
\text { water and } \\
\text { ff94 for } \\
\text { Kv1.2 }\end{array}$ \\
\hline Voltage & $\begin{array}{c}800 \mathrm{mV} \text { on } \\
S F \\
200 \mathrm{mV} \text { on } \\
N C\end{array}$ & $\begin{array}{c}160 \mathbf{~ m V} \text { on } \\
S F \\
40 \mathbf{~} \mathbf{~ V ~ o n ~} \\
N C\end{array}$ & $\begin{array}{c}800 \mathrm{mV} \text { on } \\
S F \\
200 \mathrm{mV} \text { on } \\
N C\end{array}$ & $\begin{array}{c}800 \mathrm{mV} \text { on } \\
S F \\
200 \mathrm{mV} \text { on } \\
N C\end{array}$ & $\begin{array}{c}1000 \mathrm{mV} \text { on } \\
\text { entire system }\end{array}$ \\
\hline $\begin{array}{c}\text { total } \\
\text { simulation } \\
\text { time }[\mu \mathrm{s}]\end{array}$ & 2.0 & 1.92 & 0.1 & 0.1 & 0.50 \\
\hline $\begin{array}{l}\text { independent } \\
\text { simulations }\end{array}$ & 50 & 32 & 10 & 10 & 50 \\
\hline $\begin{array}{c}\text { \# of ion } \\
\text { permeation }\end{array}$ & 204 & 10 & 11 & 10 & 94 \\
\hline $\begin{array}{l}\text { \# of water } \\
\text { permeation }\end{array}$ & 215 & 10 & 12 & 9 & 104 \\
\hline $\begin{array}{c}\text { average time } \\
\text { from } N C \text {-in to } \\
S F \text {-in [ns] }\end{array}$ & 1.9 & 10.2 & 2.0 & 1.0 & 1.2 \\
\hline $\begin{array}{l}\text { average time } \\
\text { from } S F \text {-in to } \\
N C \text {-in [ns] }\end{array}$ & 7.9 & 181.8 & 7.1 & 9.0 & 4.1 \\
\hline
\end{tabular}

Column \#1 shows the result presented in the main text. The terms in bold in columns \#2-\#5 indicate that the conditions are different from those in column \#1. Column \#2 is the simulation run using a membrane potential of $+200 \mathrm{mV}$. Fig. S1 was calculated using column $\# 2$. The water orientations shown in Fig. S8 were obtained from MD runs using the different force fields for the water and the Kv1.2 channel that are indicated in column \#3, \#4, and \#5. In all simulations, the ion-unoccupied period in the $N C$ region, which is the time from the $S F$-in moment to the $N C$-in moment, accounts for the differences in the cycle permeation time. 
Table S2. The energy change of $I_{1}$ at $S_{3}$ and its components under the effect of an ion occupying the $N C$.

\begin{tabular}{lllllllll}
\hline Total & Channel & $I_{0}$ & $I_{2}$ & other $\mathrm{K}^{+}$ & water & $\mathrm{Cl}^{-}$ & lipid & $E$ field \\
\hline$\Delta E=8.7$ & -18.7 & 31.7 & 12.1 & 1.9 & -18.0 & -0.2 & 0.0 & -0.1 \\
\hline
\end{tabular}

The energy increase, $\Delta E$, is defined as $\Delta E=E_{\text {ion-occupied }}-E_{\text {ion-unoccupied, }}$, where $E_{\text {ion-occupied }}$ is the energy during the period of the ion-occupied in the $N C$ region (from the $N C$-in moment to the $S F$-in moment) and $E_{\text {ion-unoccupied }}$ is that during the period of the ion-unoccupied in the $N C$ region (from the $S F$-in moment to the $N C$-in moment).

Table S3. The energy change of $I_{2}$ at $S_{1}$ and its components under the effect of an ion occupying the $N C$.

\begin{tabular}{lllllllll}
\hline Total & Channel & $I_{0}$ & $I_{1}$ & other $\mathrm{K}^{+}$ & Water & $\mathrm{Cl}^{-}$ & lipid & $E$ field \\
\hline$\Delta E=2.9$ & -12.8 & 15.3 & 5.4 & 4.7 & -9.3 & -0.3 & 0.0 & -0.1 \\
\hline
\end{tabular}

$\Delta E$ is defined in the same manner as in Tab. $\mathrm{S} 2$. 
Table S4. Ion permeation at $0.05,0.54$, and $0.99 \mathrm{M}$.

\begin{tabular}{llll}
\hline & $\mathbf{0 . 0 5} \mathbf{~ M}$ & $\mathbf{0 . 5 4} \mathbf{M}$ & $\mathbf{0 . 9 9} \mathbf{~ M}$ \\
\hline $\begin{array}{l}\text { simulation } \\
\text { time }[\mu \mathrm{s}]\end{array}$ & 3.17 & 0.44 & 0.35 \\
$\# \quad$ of ion & 110 & 118 & 115 \\
permeation & & & \\
$\# \quad$ of water 125 & 115 & 108 \\
permeation & & & \\
$\begin{array}{l}\text { average time } \\
\text { from } N C \text {-in to }\end{array}$ & & 1.9 & 2.0 \\
$S F$-in [ns] & & \\
$\begin{array}{l}\text { average time } \\
\text { from } S F \text {-in to }\end{array}$ & & & \\
$N C$-in [ns] & & & \\
\hline
\end{tabular}

The applied force fields and voltage are the same as the conditions during the main run (column \#1 in Tab. S1). 
Movie S1. Movie of the ion permeation through the $S F$. The red, blue, and green balls represent $I_{0}, I_{1}$, and $I_{2}$, respectively. The water molecules that intercalated between the ions are shown using the space-filling model. The backbone carbonyl of the selectivity filter $(S F)$ and the $\mathrm{OH}$-side chain of Thr347 are also shown. The sampling time was $10 \mathrm{ps}$, and the recording time was -100 ps $<\Delta t<100 \mathrm{ps}$ from the $S F$-in time. At this time resolution, $I_{0}$ seems to kick the column of ions and the water molecules in the $S F$, and $I_{2}$ leaves the $S F$.

Movie S2. Movie of the ion permeation through the $S F$ with 1 ps sampling time. The recording time was $-50 \mathrm{ps}<\Delta t<50$ ps. $I_{1}$ fluctuates between $S_{3}$ and $S_{2}$. When $S_{3}$ becomes vacant, the $I_{0}$ and the water molecule at $S_{4}$ shift downward to fill the vacant space.

Movie S3. Movie of the ion permeation through the $S F$ with 0.1 ps sampling time. The recording time was $-14.9 \mathrm{ps}<\Delta t<5.0 \mathrm{ps}$. After the downward shift of $I_{1}$, the following or chasing motions of $I_{0}$ and a water molecule are clearly seen. 